\title{
A Influência da Reforma Gerencial sobre a Cultura Organizacional no Âmbito da Gestão Escolar
}

\author{
Clarície Novaes Valgueiro Barros ${ }^{1}$, Jocimara Santos de Moura ${ }^{2}$, Niuvania Layze Muniz Brandão ${ }^{3}$, \\ Lucimara Araújo Campos Alexandre ${ }^{4}$
}

Resumo: Resumo: A reforma gerencial tentou introduzir na Administração Pública brasileira, até então marcada pelas disfunções dos modelos anteriores, princípios já aplicados pela gestão empresarial, sobretudo o foco no resultado e a busca pela excelência. $\mathrm{O}$ presente trabalho tem como objetivo, através da pesquisa bibliográfica, analisar a influência da reforma gerencial sobre a cultura organizacional na gestão escolar, identificando as ações implementadas para promover a mudança cultural bem como os principais entraves a essa implementação. Percebe-se que a implementação dos princípios da Administração Gerencial no âmbito escolar vem se desenvolvendo de forma lenta, visto a complexidade da cultura organizacional em um ambiente tradicionalista como a escola, o que ratificou a importância do incentivo a iniciativas como a participação democrática na gestão escolar e a descentralização da gestão como auxiliares na assimilação das práticas gerenciais na cultura organizacional escolar com o fim de aumentar os resultados e a melhoria da educação no Brasil.

Palavras-chave: Administração Pública. Reforma Gerencial. Cultura Organizacional. Gestão Escolar.

\section{The Influence of Management Reform on Organizational Culture in the Field of School Management}

\begin{abstract}
Management reform attempted to introduce into the Brazilian Public Administration, hitherto marked by the dysfunctions of previous models, principles already applied by business management, especially the focus on results and the search for excellence. The present work aims to analyze the influence of managerial reform on organizational culture in school management, identifying the actions implemented to promote cultural change as well as the main obstacles to this implementation. It is noticed that the implementation of the principles of the Management Administration in the school environment has been developing slowly, given the complexity of the organizational culture in a traditionalist environment such as the school, which ratified the importance of encouraging initiatives such as democratic participation in management School and the decentralization of management as auxiliaries in the assimilation of managerial practices in the school organizational culture in order to increase the results and the improvement of education in Brazil.
\end{abstract}

Keywords: Public Administration. Management Reform. Organizational Culture.School Management.

\footnotetext{
Especialista em Contabilidade Pública e Responsabilidade Fiscal pela UNINTER. Graduada em Farmácia pela Universidade Federal de Pernambuco - UFPE. Graduanda do Curso de Administração Pública da Universidade Federal do Vale do São Francisco - UNIVASF. clariciefarma@hotmail.com

2 Graduanda do Curso de Administração Pública da Universidade Federal do Vale do São Francisco - UNIVASF. jsm-mara@hotmail.com Graduanda do Curso de Administração Pública da Universidade Federal do Vale do São Francisco - UNIVASF. layzetn@hotmail.com Doutoranda em Oncologia pela Fundação Antônio Prudente - A. C. Camargo Cancer Center (UFPE). Professora Assistente do Colegiado de Enfermagem dos módulos Estágio Supervisionado I e II e da Residência Multiprofissional em Saúde da Família da Universidade Federal do Vale do São Francisco (UNIVASF). Pesquisadora do Núcleo de Estudos em Saúde Coletiva (NESCO). Orientadora no curso de Bacharelado em Administração Pública da UNIVASF. Lucimara.univasf@gmail.com
} 


\section{Introdução}

No Brasil, a Administração Pública assumiu diversas tipologias, com forte ligação existente entre a tipologia de gestão pública vigente e o contexto sociocultural à época. Conforme os ensinamentos de Silva (2013), embora se possa falar em evolução dos modelos da Administração Pública no Brasil, passando do Patrimonialista, ao Burocrático e, após, ao Gerencial, sabe-se que nenhum desses modelos foi capaz de suplantar por inteiro o anterior, havendo, atualmente, a predominância das características gerenciais com resquícios das tipologias precedentes.

Segundo Capobiango et al. (2010), as reformas administrativas podem ser compreendidas como um processo de adaptação da máquina pública ao ambiente em que se insere e se apresentam como tentativas adotadas pelo Estado para que o mesmo possa evoluir de modo a eliminar práticas com aplicações esgotadas. Entre essas reformas, destaca-se a chamada Reforma Gerencial, cujo objetivo principal, segundo Chiavenato (2008), seria flexibilizar a Administração Pública e desburocratizá-la, propondo a adoção de princípios de gestão empresarial nas máquinas estatais, em diversas regiões do mundo, assumindo, de acordo com suas origens históricas, culturais e políticas, aspectos distintos em cada uma delas.

Para Pimenta (1998), o principal fundamento para a reforma gerencial foi a crise do Estado Burocrático Brasileiro, com ações cada vez menos efetivas e a custos cada vez mais elevados. Conforme esse autor, a reforma gerencial teve como princípio fundamental dar maior autonomia de decisão ao gerente público, distanciando-se das tendências autoritárias da burocracia e consolidando a democracia também dentro das organizações públicas e não somente através do voto esporádico da população.

Segundo os ensinamentos de Costa (2008), a Administração Pública Gerencial é orientada predominantemente pelos valores da eficiência e da qualidade na prestação de serviços públicos e pelo desenvolvimento de uma cultura gerencial nas organizações. Conforme esse autor, esse modelo não nega todos os princípios da Administração Pública Burocrática, já que ambos os modelos pregam a valorização do interesse público, a meritocracia, a constante avaliação de desempenho, entre outras características convergentes. Costa (2008) afirma que a principal diferença entre o modelo Burocrático e o Gerencial está 
na forma de controle, que deixa de se basear nos processos (controle dos meios) para se concentrar nos resultados (controle dos fins).

Bresser-Pereira (2003) afirma que o objetivo da reforma gerencial foi aumentar a eficiência e a efetividade dos órgãos e agências do Estado, melhorar a qualidade das decisões estratégicas do governo e sua burocracia e assegurar o caráter democrático da Administração Pública. Coelho (2000) esclarece que a ideia norteadora dessa nova concepção de Administração Pública relaciona-se com os conceitos de eficiência, flexibilização, controle finalístico, contrato de gestão, qualidade e cidadão-cliente.

Vários estudiosos se debruçam sobre o tema das reformas, buscando desvendar os motivos que impedem o alcance dos objetivos e metas propostas. Dentre esses, Rezende (2004) lista a chamada "falha sequencial" relacionada à descontinuidade, ao abandono e ao término de processos de intervenção, antes que seus objetivos sejam alcançados ou que tenha ocorrido melhoria de desempenho no aparato burocrático. Em estudo anterior, Rezende (2002) já havia apontado diversas outras explicações na literatura, como a institucionalização, a resistência, as ondas de reformas, os conflitos de princípios, as contradições, ambiguidades e incertezas, entre outras.

Segundo Ribeiro, Pereira e Benedict (2013), as reformas da Administração Pública brasileira são criticadas por se transformarem em políticas simbólicas e não corresponderem às expectativas do povo brasileiro. Apesar disso, esses autores ratificam que as reformas contribuíram para redefinir o papel do Estado e suas relações com a sociedade, além de incentivar o debate acadêmico e profissional sobre a Administração Pública.

Pontes (2007) considera a escola como um espaço preservado para a formação do cidadão e referência da comunidade, bem como um ambiente de convívio, segurança e paz, onde se espera que seja um local estruturado e preparado para formar o cidadão para o futuro, para cidadania e fortalecer a esperança de que os objetivos, sonhos e metas se tornarão realidade.

No âmbito das organizações, Fleury (1990, p. 22) define a cultura organizacional como:

Um conjunto de valores e pressupostos básicos expressos em elementos simbólicos, que em sua capacidade de ordenar, atribuir significações, construir a identidade organizacional, tanto agem como elemento de comunicação e consenso, como ocultam e instrumentalizam as relações de dominação. 
Conforme os ensinamentos de Silva (2007), entende-se por cultura da organização escolar as manifestações de valores, crenças e padrões de comportamento próprios do coletivo da instituição. A autora afirma ainda que as escolas assumem uma cultura própria em função de suas práticas e condutas, que nem sempre são consentâneas com o projeto de educação que se consolida no país. Rosa (2004) explica que a cultura escolar é desenvolvida ao longo do tempo, raramente antes de decorridos dez anos de funcionamento da instituição, que é o tempo necessário para que os padrões de comportamento sejam internalizados pelas pessoas que compõem a escola.

No caso das organizações escolares, a cultura reflete o espelho da diversidade grupal, por serem formadas por grupos de supervisores, colaboradores, diretores, alunos, entre outros, com perfis diversos, tanto do ponto de vista cognitivo quanto demográfico (idade, sexo e raça ou etnia), o que torna tais organizações multiculturais e bastante complexas. Pontes (2007) afirma que as organizações escolares são regidas por uma cultura organizacional e valores específicos, com objetivos de transmitir e produzir conhecimentos.

Para Capobiango et al. (2010), cada país apresenta um perfil peculiar, com suas limitações e suas características culturais, as quais devem ser levadas em consideração ao se propor alguma alteração que interfira na vida social de todos que compõem o Estado. Nesse sentido, o impacto das mudanças também pode variar entre os mais diversos setores da economia dentro do país, sofrendo fortes resistências naqueles setores marcados por uma cultura organizacional mais tradicionalista como, por exemplo, na educação.

Conforme Capobiango et al. (2010), a mudança organizacional é um assunto desafiador, seja pela sua profundidade e complexidade, seja pela variedade de enfoques existentes, devendo-se o gestor considerar o fato de que o ambiente, por si só, não provoca mudanças dentro da organização; são as pessoas que criam novos rumos e cursos estratégicos. Ainda segundo esses autores, a mudança organizacional deve ser entendida e trabalhada não somente sob a ótica de estratégias, processos ou tecnologias, mas como uma mudança de relações, do indivíduo com a organização, dele com seus pares, da organização com a sociedade, do indivíduo com a sociedade e dele consigo mesmo.

A educação, em seu contexto mais amplo, é capaz de formar cidadãos conscientes de seus direitos e deveres, aptos a contribuírem para o desenvolvimento da sociedade como um 
todo. Além de propulsor do desenvolvimento individual e social, a educação tem-se mostrado essencial para a evolução, não apenas cognitiva do educando, mas também da capacidade de formar opinião e exercer plenamente a cidadania.

Conforme Faro (2007), a educação é uma condição indispensável para que se produza um homem histórico-socialmente construído, formado para bem viver em sociedade e para a realização da liberdade. Segundo Vasconcelos (2007), a escola fornece um horizonte mais amplo no qual a criança ou o jovem inscrevem as suas vidas. Daí a importância de uma educação com responsabilidade e compromisso. Vasconcelos (2007) ressalta que a escola pode ser vista como um importante agente de mudança e fator de desenvolvimento social, sobretudo de valores como solidariedade, justiça, tolerância, respeito e sabedoria.

Em tempos de escassez de recursos, sobretudo o atual, as escolas públicas e privadas tem feito grande esforço, através da implantação de novos modelos de gestão, a fim de obterem resultados, ainda que em tempos de crise. Conforme Luck (2009), a gestão escolar constitui uma das áreas de atuação profisssional na educação destinada a realizar o planejamento, a organização, a liderança, a orientação, a mediação, a coordenação, o monitoramento e a avaliação dos processos necessários à efetividade das ações educacionais orientadas para a promoção da aprendizagem e formação dos alunos.

Assim como as organizações públicas e privadas estão buscando novos paradigmas a fim de acompanhar o ambiente dinâmico e sobreviver às novas demandas, sobretudo as impostas pela globalização e pelo avanço da tecnologia da informação e comunicação, a gestão educacional, conforme Castro (2008), vem assumindo uma nova configuração, pautada pela noção de modernidade e a ideia de que é necessário superar os antigos modelos centralizadores de gestão, não mais adequados às novas configurações do mundo do trabalho.

Conforme assinalado por Castro (2008), no campo da gestão educacional, fica evidenciada a necessidade de se produzir uma transformação profunda na gestão educativa tradicional, que permita articular, efetivamente, a educação com as demandas econômicas, sociais, políticas e culturais, rompendo com o isolamento das ações educativas, bem como de impulsionar o processo de descentralização, de regionalização e de desconcentração, através do desenho de mecanismos mais ágeis de avaliação de resultados, de programas eficazes de compensação educativa e de sistemas de informação e investigação para a tomada de decisão, como fórmulas destinadas a aumentar a capacidade de gestão. 
Consoante Souza e Ribeiro (2017), o mundo permanece em constantes e rápidas modificações, motivo pelo qual a escola não pode estar à margem ou em outra dimensão se não a da mudança, embora, muitas vezes, seja uma instituição com tendências mais conservadoras. Por essa razão, faz-se necessária a incorporação dos ideais da administração gerencial no âmbito escolar de forma a permitir não só o cumprimento das determinações legais, mas também a consecução dos urgentes anseios sociais quanto à melhoria da educação brasileira.

Paro (1987) reflete a necessidade de relativizar e avaliar os aspectos da ação empresarial que, de fato, devem ser introduzidos no espaço escolar. No entanto, Silva (2007), avalia como positiva a tendência da inserção da gestão gerencial na Educação Pública, sobretudo nos aspectos financeiros. Souza e Ribeiro (2017) afirmam que a gestão educacional, em seus moldes gerenciais, apresentará características em direção à redução de custos, melhorias na qualidade dos serviços prestados, presença de modelos de avaliação de desempenho, entre outras, mais sensíveis às necessidades educacionais das escolas do período contemporâneo, como dinâmica e criatividade, iniciativa, cooperação, motivação e capacidade interpretativa das problemáticas.

Estudar as influências da reforma gerencial sobre a cultura organizacional e a gestão escolar, justifica-se pela necessidade de se compreender as oportunidades e fragilidades obtidas na execução desse processo, visando a apontar alternativas na busca da eficiência e eficácia organizacional na gestão escolar e a satisfação do cidadão.

Uma vez que a cultura organizacional influencia diretamente os servidores na sua maneira de pensar, agir e até mesmo na realização de uma tarefa, o estudo em tela buscou, através da pesquisa bibliográfica, verificar até que ponto as características culturais podem atuar como obstáculos no esforço de promover mudanças e introduzir inovações, sobretudo no âmbito escolar, em geral extremamente tradicional e resistente a grandes reformas.

No intuito de alcançar este objetivo geral, estabeleceram-se os seguintes objetivos específicos: Destacar a influência da reforma gerencial sobre a cultura organizacional na gestão escolar; Identificar as ações implementadas para promover a mudança cultural proposta pela reforma gerencial da Administração Pública brasileira e os principais atores envolvidos; Identificar os principais entraves à implementação do modelo gerencial em uma escola pública. 


\section{Métodos}

De acordo com Zanella (2012), a pesquisa bibliográfica fundamenta-se a partir do conhecimento disponível em fontes bibliográficas, principalmente livros e artigos científicos. Segundo Köche (1997, p. 122), esse tipo de pesquisa tem a finalidade de ampliar o conhecimento na área estudada, com o objetivo de depois utilizá-lo como modelo teórico que dará sustentação a outros problemas de pesquisa.

Zanella (2012) adverte que a pesquisa bibliográfica não se restringe ao campo de atuação no levantamento e na discussão da produção bibliográfica existente sobre o tema, de forma que, conforme os ensinamentos de Gil (2007, p. 60), o processo de pesquisa deve envolver a escolha do tema, o levantamento bibliográfico preliminar, a formulação do problema, a elaboração do plano provisório de assunto, a busca das fontes, a leitura do material, o fichamento, a organização lógica do assunto e, por fim, a redação do texto.

O presente estudo adotou como estratégia metodológica a pesquisa bibliográfica, na qual foram utilizados artigos científicos, bem como livros sobre o assunto. Todos os 19 (dezenove) artigos científicos, selecionados a partir da variável de interesse, foram obtidos através da base de dados Google Acadêmico, a qual, segundo Puccini et. al. (2015), tem se projetado como um dos principais portais de pesquisa, uma vez que o resgate de artigos científicos é feito por toda a internet, teoricamente sem limites, além de ser simples e prático. Foram utilizados como descritores para a pesquisa: Reforma Gerencial; Reforma Administrativa; Administração Pública Gerencial; Gestão Escolar; Cultura Organizacional.

Após a coleta dos dados, procedeu-se à leitura crítica de todo o arcabouço teórico obtido, a fim de ampliar o conhecimento acerca do tema em questão.

\section{Referencial Teórico}

A maioria dos trabalhos sobre as reformas mais recentes se encarrega de recuperar os principais antecedentes desses processos de transformação. Existem, hoje, inúmeros relatos, análises e estudos sobre as experiências de Reforma da Administração Pública Federal Brasileira, com diferentes abordagens, graus de profundidade e níveis de implicação com os 
projetos. Pode-se verificar que, embora existam inúmeros estudos sobre as reformas administrativas, a grande maioria foca em apenas descrever suas características em um ambiente ideal e homogêneo, sem avaliar os seus efeitos em áreas específicas da gestão pública, com características certamente diversas, como a educação, a saúde, a segurança, a fiscal, dentre outras (COSTA, 2008).

Entre os diversos entraves verificados para a efetivação dos princípios do paradigma Gerencial no ambiente escolar nas pesquisas levantadas, destacam-se a falta de autonomia na resolução dos problemas, a falta de concatenação entre as normas e regulamentos dos órgãos de gestão com a realidade e com o princípio da eficácia (preocupando-se muitas vezes com resultados quantitativos, como o número de alunos matriculados, aprovados ou evadidos), excesso de normas, falta de incentivos materiais para órgãos pedagógicos, administrativos, e outros cargos de apoio com competência no auxílio, excesso de responsabilidade sobre o órgão de direção (ROSA, 2004).

É importante ressaltar que, apesar do excesso de responsabilização do gestor escolar pela identidade cultural da organização, o delineamento dessa cultura é multifatorial, visto ser influenciada por manifestações de valores, crenças e padrões de comportamento próprios do coletivo da instituição e daqueles que o compõe. Assim, a cultura organizacional, dinâmica, consegue convergir internamente as subculturas dos integrantes da instituição e distingue a instituição das demais, conforme os ensinamentos de Silva (2007).

Nesse sentido, Santos Filho (1999, p. 35) considera que a cultura organizacional é um mecanismo que tem por objetivo "conformar condutas, homogeneizar maneiras de pensar e viver a organização, introjetar uma imagem de que todos são iguais, escamoteando as diferenças e anulando a reflexão", alertando para o equívoco de interpretar as resistências à tentativa de mudança cultural como mero reflexo da natureza humana e não como um choque de interesses. Em outras palavras, a cultura organizacional, formada por diversas identidades particulares, por vezes conflitantes, não raras vezes oferece às reformas administrativas um campo hostil e avesso às mudanças.

Apesar das dificuldades verificadas na implantação dos ideais gerenciais no âmbito escolar, verificou-se que a gestão democrática dentro da escola pode ajudar na mudança de paradigma e melhorar a eficiência do serviço prestado à comunidade.

De acordo com a UNICEF (2004), a gestão escolar democrática funda-se no 
compartilhamento de decisões e informações, na preocupação com a qualidade da educação e com a relação custo-benefício e na transparência, compartilhando decisões com pais, alunos, professores, funcionários e outras pessoas da comunidade na administração escolar através dos chamados "Conselhos Escolares", já presentes em muitas escolas do país, bem como através das reuniões pedagógicas, festas, exposições e apresentações dos alunos, entre outros mecanismos de participação conjunta. Infelizmente, a gestão democrática ainda é um desafio às escolas, uma vez que a democracia no processo educativo é relativamente recente, assim como o processo de democratização política em geral.

\section{A Influência da Reforma Gerencial sobre a cultura organizacional na gestão escolar}

A cultura organizacional tem sido caracterizada por vários autores, segundo Pettigrew (1979), introduziu o conceito antropológico de cultura e mostrou como conceitos relacionados com: simbolismo, mito e rituais pode ser usado na análise organizacional; Dandridge, Mitroff e Joyce (1980) mostraram como o estudo destes mitos e símbolos ajudam a revelar a estrutura de uma organização. Assim como alguns autores caracterizam cultura organizacional, existem também os que definem clima organizacional; Para Bennis (1996, p.6), "clima significa um conjunto de valores ou atitudes que afetam a maneira pela qual as pessoas se relacionam uma com as outras, tais como: sinceridade, padrões de autoridade, relações sociais, etc."

A cultura organizacional e o seu clima organizacional podem ser influenciados tanto por fatores internos (da própria escola) como fatores externos (ambiente). Na concepção de gerencialismo, o objetivo da gestão escolar é aumentar a eficiência e eficácia dos seus gestores, fatores que se resultariam em indicadores de desempenho e resultados. Para a obtenção desses indicadores o fundamental aspecto assinalado é a mudança na cultura organizacional. A ideia da reforma gerencial é que a atividade gestora se paute na inovação, na criatividade de todos os seus segmentos.

De acordo com Castro, 2007, p. 131:

Esse novo paradigma gerencial vem substituindo gradativamente os modelos burocráticos de gestão, trazendo modificações em todos os setores sociais. No campo da educação, desde os sistemas centrais até à escola, passam a ser adotadas diretrizes gerenciais na condução dos serviços educacionais. As influencias fazem-se 
presentes na gestão escolar, entre outros, por meio da descentralização; na instituição dos colegiados; e na participação da comunidade escolar nos processos de decisão da escola.

\section{Ações Implementadas para Promover a Mudança Cultural Proposta pela Reforma Gerencial da Administração Pública Brasileira}

A reforma gerencial no Brasil teve como marco histórico o ano de 1995, com a publicação, nesse ano, do Plano Diretor da Reforma do Estado e o envio para o Congresso Nacional do projeto de emenda à Constituição Federal n. 19/1998, conhecida como Emenda da Reforma Administrativa. Tal reforma tentava romper com as disfunções do modelo burocrático, trazendo para a gestão pública ideais já sedimentados na gestão empresarial, como a busca da eficiência, a satisfação do cidadão (agora visto como cliente por patrocinar os serviços públicos como contribuinte) e, sobretudo, o foco no resultado.

No campo educacional, são perceptíveis as mudanças que ocorreram na gestão educacional, a partir do novo modelo gerencial adotado para os serviços públicos. A própria legislação educacional se encarregou de normatizar as ações da gestão educacional, para possibilitar uma gestão mais descentralizada, mais ágil e mais participativa. Embora a Constituição Federal de 1988 e a Lei de Diretrizes e Bases da Educação Nacional (Lei n. 9.394/96) se refiram à gestão democrática, os programas e projetos desenvolvidos pelos últimos governos se alinham, de fato, a uma perspectiva de gestão gerencialista. Entre eles podem-se citar o Plano de Desenvolvimento da Educação (PDE), o Plano Estratégico das Secretais (PES) e o Plano de Desenvolvimento da Escola (PDE-Escola).

\section{Principais Entraves à Implementação do Modelo Gerencial em uma Escola Pública}

Dentre os principais entraves, à modernização da gestão pública destaca-se a mudança cultural, por existir em muitas organizações, vestígios de modelos de administração patrimonialista e burocrática. Percebe se ainda a ausência de conhecimento relacionado a gestão estratégica e de práticas administrativas modernas. Isso nos mostra a carência de capacitação dos servidores, a importância da administração gerencial a busca de práticas de 
gestão e o entendimento de seus benefícios é que os gestores poderiam se convencer da importância de um novo modelo de gestão e buscar sua implantação.

\section{Considerações Finais}

Diante do exposto, este ensaio teórico, elaborado com base em referências bibliográficas, buscou contextualizar o cenário da reforma gerencial da Administração Pública Brasileira, suscitando indagações e reflexões sobre o tema, sem ter, no entanto, a pretensão de esgotar o assunto. Para tanto, foram selecionados diversos trabalhos científicos acerca do paradigma da Administração Pública Gerencial, cultura organizacional e ambiente escolar.

Entre as dificuldades encontradas, pode-se destacar a vastidão da bibliografia acerca do modelo gerencial de Administração Pública, a Reforma Gerencial e a cultura organizacional e, em contrapartida, a escassez de estudos que congreguem esses temas no âmbito escolar.

A partir da análise da bibliografia selecionada, percebe-se que a implementação dos princípios da Administração Gerencial no âmbito escolar vem se desenvolvendo de forma paulatina, com incentivos dos governos e, principalmente, da participação democrática na gestão escolar, o que sugere que novas iniciativas, sobretudo às voltadas para a descentralização da gestão escolar e o aumento da autonomia dos seus gestores, podem auxiliar na assimilação das práticas gerenciais na cultura organizacional escolar, aumentando os resultados e a melhoria da educação no Brasil.

Por fim, espera-se que as informações aqui descritas possam embasar teoricamente outros trabalhos científicos e responder questões levantadas sobre o tema, além de servir de incentivo para que outros pesquisadores aprofundem seusconhecimentos acerca do Paradigma Gerencial na Gestão Escolar. 
Id on Line Revista Multidisciplinar e de Psicoloqia

Id on Line Multidisciplinary and Psycology Journal

\section{Referências}

BENNIS, W. A Formação do Líder. São Paulo: Atlas, 162 p, 1996.

BRASIL. Constituição (1988). Constituição da República Federativa do Brasil. Brasília, DF: Senado Federal, 1988.

BRASIL. Congresso Nacional. Lei n. 9.394, de 20 de dezembro de 1996, que estabelece as diretrizes e bases da educação nacional. Brasília, DF, 1996.

BRESSER-PEREIRA, L. C. Da Administração Pública Burocrática à Gerencial. In: BRESSER PEREIRA, L. C.; SPINK, P. Reforma do Estado e Administração Pública Gerencial. 5. ed. Rio de Janeiro: Ed. FGV, 2003.

CAPOBIANGO, R. P. et al. Reformas Administrativas no Brasil: uma abordagem teórica e crítica. In: ENCONTRO DE ADMINISTRAÇÃO PÚBLICA E GOVERNANÇA, 2010, Vitória-ES. $\quad$ Artigo... Vitória: $\quad$ ANPAD, $2010 . \quad$ Disponível em< $<$ http://www.anpad.org.br/diversos/trabalhos/EnAPG/enapg_2010/2010_ENAPG116.pdf. $>$ Acesso em: 10 set. 2016.

CASTRO, A. M. D. A. Administração Gerencial: a nova configuração da gestão da educação na América Latina. Revista Brasileira de Política e Administração da Educação, v.24, n.3, p. 389-406, set./dez. 2008. Disponível em <http://seer.ufrgs.br/rbpae/article/view/19265>. Acesso em 31 mar. 2017.

CASTRO, A. M. D. A. Gerencialismo e Educação: estratégias de controle e regulação da gestão escolar. In, CABRAL NETO, A. et al(orgs.). Pontos e contrapontos da política educacional: uma leitura contextualizada de iniciativas governamentais. Brasília:DF, Líber Livro Editora, 2007. p.131

CHIAVENATO, I. Administração Geral e Pública. 2.ed. Rio de Janeiro: Elsevier, 2008.

COELHO, D. M. Elementos Essenciais ao Conceito de Administração Gerencial.Revista de Informação Legislativa. Brasília, ano 37, n. 147, jul./set., 2000. Disponível em $<$ http://scholar.googleusercontent.com/scholar?q=cache:YosJOgCd Z0J:scholar.google.com/ + administra $\% \mathrm{C} 3 \% \mathrm{~A} 7 \% \mathrm{C} 3 \% \mathrm{~A} 3 \mathrm{o}+$ gerencial + conceito\&hl $=\mathrm{pt}-\mathrm{BR} \& \mathrm{as} \_\mathrm{sdt}=0,5>$. Acesso em 20 abr. 2017.

COSTA, F. L. História das Reformas Administrativas no Brasil: narrativas, teorizações e representações. Revista do Serviço Público, Brasília, v. 59, n. 3, p. 271-288, 2008.

DANDRIDGE, T., MITROFF, I.; JOYCE, W. Organizational Symbolism: a topic to expand organizational analysis. Academyof Management Review, Vol. 5, pp. 248-56, 1980.

FARO, V. H. Gestão Escolar, Democracia e Qualidade do Ensino. São Paulo: Ática, 2007. 
FLEURY, M.T. L. O desvendar a cultura de uma organização: uma discussão metodológica. In: FLEURY, M. T. L.; FISCHER, R.M. Cultura e poder nas organizações. São Paulo: Atlas, 1990.

FUNDO DAS NAÇÕES UNIDAS PARA A INFÂNCIA. Indicadores da Qualidade na Educação. UNICEF, PNUD, Inep-MEC (coordenadores). São Paulo: Ação Educativa, 2004.

GIL, A. C. Métodos e Técnicas de Pesquisa Social. 5. ed. São Paulo: Atlas, 2007.

KÖCHE, J. C. Fundamentos de Metodologia Científica: teoria da ciência e prática da pesquisa. 14. ed. rev. amp. Petrópolis, RJ: Vozes, 1997.

LUCK, H. Dimensões da Gestão Escolar e suas Competências. Curitiba: Editora Positivo, 2009.

NETO, A. C.; CASTRO, A. M. D. Araújo. Gestão Escolar em Instituições de Ensino Médio: entre a gestão democrática e a gerencial. Educ. Soc., Campinas , v. 32, n. 116, p. 745-770, Sept. 2011 . Disponível em <http://www.scielo.br/scielo.php?script=sci_arttext\&pid=S0101$73302011000300008 \& \operatorname{lng}=$ en\&nrm=iso>. Acesso em 07 ago. 2017.

PARO, V. H. Administração escolar: introdução crítica. 2. ed. São Paulo: Cortez; Autores Associados, 1987.

PETTIGREW, A.M. On Studying Organizational Cultures. Administrative Science Quarterly, v. 24, p. 570-81, 1979.

PIMENTA, C. C. A Reforma Gerencial do Estado Brasileiro no Contexto das Grandes Tendências Mundiais. Revista de Administração Pública, Rio de Janeiro, 32(5), p. 173-199, set./out., $1998 . \quad$ Disponível em

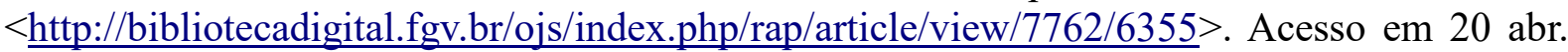
2017.

PONTES, R. N. (coord.); CRUZ, C. R. R; MELO, S. M. Relações Sociais e Violências nas Escolas. Belém: UNAMA, 2007.

PUCCINI, L. R. S. et al. Comparativo entre as Bases de Dados PubMed, SciELO e Google Acadêmico com o Foco na Temática Educação Médica. Cadernos Unifoa, Volta Redonda, ed. 28, p. 75-82, ago. 2015. Disponível em <web.unifoa.edu.br/cadernos/edicao/28/7582.pdf>. Acesso em 24 jul. 2017.

REZENDE, F. da C. O dilema do Controle e a falha sequencial nas reformas gerenciais. Revista do Serviço Público, v. 53, n.3, 2002.

Por que falham as reformas administrativas. Rio de Janeiro: FGV, 2004. 
RIBEIRO, L. M. de P.; PEREIRA, J. R.; BENEDICT, G. C. As Reformas da Administração Pública Brasileira: Uma Contextualização do seu Cenário, dos Entraves e das Novas Perspectivas. In: ENCONTRO DA ANPAD, 37., 2013, Rio de Janeiro. Anais... Rio de Janeiro: ANPAD, 2013. p. $1-15$.

ROSA, C. Gestão Estratégica Escolar. Petrópolis: Vozes, 2004.

SANTOS FILHO, H. Cultura Organizacional: uma análise crítica. Salvador: Memorial das Letras, 1999.

SILVA, A. do C. Evolução da Administração Pública no Brasil e Tendências de Novos Modelos Organizacionais. Cuiabá: ICE, 2013. Disponível em < www.ice.edu.br/TNX/storage/webdisco/.../27b4d512efbac7d37520bc37aa78cac1.pdf>.

Acesso em 08 set. 2016.

SILVA, G. R. da. Tendências Actuais na Formação em Administração Educacional. Rev. Port. de Educação, v. 20 n.1 Braga jan. 2007, p. 221-245.

SOUZA, L. D. M.; RIBEIRO, M. S. de S. O Perfil do Gestor Escolar Contemporâneo: das permanências às incorporações para exercício da função. Espaço do Currículo, v.10, n.1, p. 106-122, Janeiro a Abril de 2017. Disponível em $<$ http://periodicos.ufpb.br/ojs2/index.php/rec/article/view/33386/17435>. Acesso em 28 jun. 2017.

VASCONCELOS, T. A Importância da Educação na Construção da Cidadania. Revista Saber (e) Educar, n. 12. Porto/Portugal: Escola Superior de Educação de Paula Frassinetti, 2007. Disponível em <http://repositorio.esepf.pt/handle/20.500.11796/714>. Acesso em 09 set. 2016.

ZANELlA, L. C. H. Metodologia de Estudo e de Pesquisa em Administração. 2. ed. Florianópolis: Departamento de Ciências da Administração/UFSC, 2012. 164p.

Como citar este artigo (Formato ABNT):

BARROS, Clarície N.V.; MOURA, Jocimaria S. de; BRANDÃO, Niuvania L.M.; ALEXANDRE, Lucimara A.C. A Influência da Reforma Gerencial sobre a Cultura Organizacional no Âmbito da Gestão Escolar. Id on Line Revista Multidisciplinar e de Psicologia, Maio de 2017, vol.11, n.37, p.66-79. ISSN: 1981-1179.

Recebido: 01.08.2017;

Aceito: 09.08.2017 